\title{
(Des)conexión durante el viaje turístico: uso de smartphones por parte de viajeros independientes en la ciudad de Guayaquil
}

Recibido: 21/03/17 - Aceptado: 26/04/17

\author{
Carla Ricaurte-Quijano* \\ Alejandra Arellano Agusto \\ Katherine Naranjo Salavarria \\ Facultad de Ingeniería Marítima, Ciencias \\ Biológicas, Oceánicas y Recursos Naturales \\ Escuela Superior Politécnica del Litoral, ESPOL
}

\section{RESUMEN}

A partir del análisis cualitativo de entrevistas semiestructuradas, se identifica cómo los viajeros independientes que visitan la ciudad de Guayaquil utilizan su teléfono inteligente y cómo su uso influye en su propia experiencia de viaje. Los resultados indican que los turistas entrevistados en Guayaquil usan el teléfono inteligente para comunicarse con personas, principalmente de su lugar de origen y para buscar información. Respecto a la experiencia de viaje, los participantes relacionaron el empleo de su teléfono inteligente en el destino con sentimientos de seguridad, comodidad y placer: seguridad porque los visitantes pueden identificar con precisión dónde están; comodidad porque pueden conjugar varias funciones como comunicación, cámara de fotos y música en un solo aparato, lo que disminuye la cantidad de cosas que se debe traer en el equipaje; y placer, ya que poder interactuar con familiares y amigos para compartir cada etapa del viaje contribuye a construir experiencias positivas del mismo.

Palabras clave: Teléfono inteligente, turismo, experiencia de viaje, aplicaciones móviles, Guayaquil.

*Correos electrónicos: cricaurt@espol.edu.ec·acarella@espol.edu.ec·katdnara@espol.edu.ec

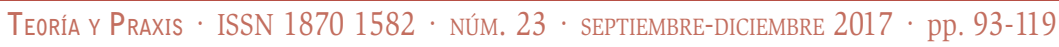




\title{
(Dis)connection during a tourism trip: The use of smartphones by independent travelers in the city of Guayaquil
}

Recieved: 21/03/17 - Accepted: 26/04/17

\begin{abstract}
Through a qualitative analysis of semi-structured interviews, this study analyses the use of smartphones by independent travelers in the city of Guayaquil and how this use influences their tourist experience. The results indicate that tourists interviewed in Guayaquil use their smartphones to communicate mainly with people in their places of origin and to look for information. In terms of their travel experience, participants associate the use of their smartphones in the destination with a sense of security, comfort and pleasure: security because they are able to know exactly where they are, comfort since one piece of equipment provides many different functions like communication, camera and music reducing the amount of equipment they have to carry with them, and pleasure since they can interact with family and friends to share every stage of their trip generating positive travel experiences.
\end{abstract}

Carla Ricaurte-Quijano*

Alejandra Arellano Agusto

Katherine Naranjo Salavarria

Facultad de Ingeniería Marítima, Ciencias

Biológicas, Oceánicas y Recursos Naturales

Escuela Superior Politécnica del Litoral, ESPOL

KEY woRDs: Smartphone, tourism, travel experience, mobile apps, Guayaquil.

*E-mails: cricaurt@espol.edu.ec·acarella@espol.edu.ec·katdnara@espol.edu.ec 


\section{Introducción}

Una de las transformaciones más fuertes que ha sufrido la industria del turismo en el siglo xxi tiene que ver con la masificación del uso del smartphone o teléfono inteligente (Florido-Benítez, 2016). Los teléfonos inteligentes son equipos con sistemas operativos avanzados que proporcionan acceso portátil a internet y cuentan con una variedad de utilidades adicionales, como cámara de fotos y video, grabadora de voz, GPS, alarma, agenda, correo electrónico y otras aplicaciones (Wang y Fesenmaier, 2013). El uso de smartphones es de dimensión mundial: solo en Estados Unidos alcanza $64 \%$ de la población (Pew Research Center, 2016). Adicionalmente, economías en rápido desarrollo, como China e India, se están convirtiendo en los mayores compradores de teléfonos inteligentes en el mundo (mobiThinking, 2014); al mismo tiempo se convierten en los países emisores de viajeros con mayor crecimiento en el mercado global (United Nations World Tourism Organization [unwTo], 2016). Según el Instituto Nacional de Estadística y Censos (INEc, 2016) más de tres millones de ecuatorianos poseen un teléfono inteligente. La masificación del uso de teléfonos inteligentes en el mundo ha significado también un aumento del uso de estos dispositivos para facilitar los viajes y el turismo (Wang, Xian y Fesenmaier, 2014). Un estudio del sitio web de viajes TripAdvisor (2015), indica que $42 \%$ de los viajeros en todo el mundo usan su teléfono inteligente para planificar y reservar su viaje y, de estos, 72 \% usa su teléfono inteligente para hacer consultas de facilidades y atracciones turísticas una vez que ya están en el destino.

Guayaquil es el primer municipio en el Ecuador en realizar un proyecto de Ciudad Digital (GAD Municipal de Guayaquil, 2014). Aurigi (2005) define a la ciudad digital como el uso de tecnologías de información para fomentar el desarrollo económico de las ciudades por medio de la comunicación con los ciudadanos y visitantes. La idea de ciudad digital también abarca los esfuerzos orientados a mejorar la prestación de servicios urbanos, incluyendo educación y salud, así como fomentar la participación ciudadana y apoyar a procesos democráticos. Para Aurigi (2005), la ciudad digital complementa y revitaliza el espacio físico urbano al extender la ciudad hacia el ciberespacio. En Guayaquil, el proyecto de Ciudad Digital incluye facilitar el acceso de la ciudadanía a internet mediante la instalación de 6000 puntos de conexión wifi, la entrega de tabletas digitales a los bachilleres de la ciudad, la instalación de kioscos digitales para 
(Des)conexión durante el viaje turístico: uso de smartphones por parte de viajeros independientes en la ciudad de Guayaquil

realizar trámites municipales y, finalmente, el desarrollo y puesta en marcha de la aplicación móvil "Guayaquil es mi destino", en la que pueden consultarse los diferentes atractivos e información turística general de la ciudad (El Universo, 22 de julio y 3 de agosto del 2015).

Mientras que el desarrollo de aplicaciones móviles para turismo sí ha sido un área de interés en el contexto académico latinoamericano (véase por ejemplo Benítez Victoria y Bermúdez Moreno, 2015; Cuadro Guerrero y Ruiz Vergara, 2017; Mariño Arias, 2012), la información e investigación relacionada con el uso del smartphone en las diferentes etapas del viaje turístico es considerablemente menor. El presente estudio se enmarca, por un lado, en el aumento del uso del smartphone por parte de los viajeros internacionales y, por otro, en el interés de los destinos latinoamericanos por promover su desarrollo turístico digitalmente, entre ellos la ciudad de Guayaquil. Por lo tanto, se pretende caracterizar el uso del teléfono inteligente por parte de los viajeros independientes que visitan Guayaquil e identificar cómo los participantes relacionan su propia experiencia de viaje con el uso del dispositivo. El estudio contribuye a identificar los usos, así como las emociones asociadas al uso del teléfono inteligente y cómo estas modifican de manera continua la relación entre el viajero y el destino; de este modo se pretende contribuir al futuro desarrollo de aplicaciones móviles y portales de turismo con especial énfasis en el contexto local y regional.

\section{La influencia del smartphone y las nuevas tecnologías en la experiencia de viaje}

Un viaje es un proceso de decisiones en el cual los viajeros permanentemente construyen y reconstruyen su experiencia de viaje mediante el aprendizaje, las emociones y los sentimientos generados por y en el lugar de destino (Jennings y Weiler, 2006). En otras palabras, cada desplazamiento de una persona fuera de su entorno habitual genera a su vez una experiencia para el viajero. Jennings y Weiler (2006), explican la experiencia de viaje recurriendo a una perspectiva lineal de tiempo compuesta por tres partes: la primera es la fase anticipatoria, en la cual se genera la expectativa de viaje, las emociones y las motivaciones del viaje. Es también la etapa de planificación del viaje. Después viene la fase experimental, que transcurre durante el viaje y comprende las actividades e 
interacción con el destino y, por último, está la fase reflexiva, que consiste en el análisis y la valoración de la experiencia de viaje, una vez que este ha concluido. Estas etapas coinciden con la propuesta de Gretzel, Fesenmair y O’Leary (2006), en la que dividen la experiencia turística en las etapas de preconsumo, consumo y posconsumo, cada una con actividades definidas. Así, en la etapa de preconsumo, el turista principalmente busca información y toma decisiones. En la de consumo se vive la experiencia y se llevan a cabo las transacciones en el lugar de destino y en el posconsumo se organizan las experiencias, se comparten y se reviven memorias y emociones.

Wang y Fesenmaier (2013), por su parte, describen la experiencia de viaje como la relación entre una actividad relacionada con el viaje en el lugar de destino, y las emociones y sensaciones que esta actividad genera en los viajeros en un tiempo y lugar determinados. En su estudio, los autores concluyen que con el uso de tecnologías de información, y en concreto de teléfonos inteligentes, se acortan o eliminan las etapas pre y posviaje, mientras se extiende la etapa de consumo, ya que, ayudados por la tecnología, los turistas investigan menos antes de viajar y buscan información durante el viaje, permitiendo la toma de decisiones de manera espontánea en los lugares de destino. De la misma forma, se comparte información y fotos con familiares y amigos mientras se está viajando, lo que reduce la etapa de posconsumo.

Investigaciones previas (Dickinson et al., 2014; Wang, Park y Fesenmaier, 2012; Wang y Fesenmaier, 2013; Wang et al., 2014, 2016) señalan que la experiencia de viaje es influida por las nuevas tecnologías y estas determinan que los impactos sean tanto positivos como negativos. Estudios que abordan el tema del uso del teléfono inteligente para viajes y turismo han tenido una tendencia a enfocarse en aspectos específicos, como el desarrollo de aplicaciones para viaje (Benítez Victoria y Bermúdez Moreno, 2015; Cuadro Guerrero y Ruiz Vergara, 2017; Dickinson et al., 2014; Mariño Arias, 2012; Wang y Xiang, 2012), la identificación del perfil de los usuarios de teléfonos inteligentes (Okazaki, Campo, Andreu y Romero, 2015; Vallespín Arán, Molinillo y Muñoz-Leiva, 2015), o el impacto del teléfono inteligente en ciertos aspectos de la experiencia turística (Dickinson et al. 2014; Tussyadiah y Zach 2012; Wang et al., 2012; Wang y Fesenmaier, 2013; Wang et al., 2014, 2016). En general, los resultados señalan cómo el uso del teléfono inteligente tiene repercusiones amplias, 
(Des)conexión durante el viaje turístico: uso de smartphones por parte de viajeros independientes en la ciudad de Guayaquil

modificando en gran medida los patrones de consumo y de comportamiento del turista, así como la forma en que se viven y se comparten las experiencias (Munar y Jacobsen, 2014), cambiando incluso el mismo significado del viaje (Wang et al., 2014).

Wang y Fesenmaier (2013) analizan la experiencia de viaje a partir de los comentarios que los turistas realizan en las aplicaciones más usadas. Los resultados identifican catorce maneras de cómo el teléfono inteligente soluciona problemas de los viajeros en temas de búsqueda de información, flexibilidad en el viaje y al compartir información y emociones. Según sus resultados, el uso del teléfono inteligente influye y cambia el comportamiento, los patrones de consumo, el estado de ánimo del turista, así como la forma en que el turista reflexiona sobre su propia experiencia. Partiendo de Jansson (2007) y Moores (2005), Wang et al. (2013), describen estos impactos como decapsulación: argumentan, por ejemplo, que las claras descripciones y la amplia información obtenida en internet podrían de cierto modo destruir el sentido de aventura del viaje, mientras que las conexiones con la vida ordinaria a través de emails o textos pueden romper el sentimiento de escape, borrando los límites entre origen y destino, tiempo de trabajo y de ocio. Sus hallazgos enfatizan cómo las tecnologías móviles pluralizan el tiempo y los espacios sociales. En este sentido, los autores indican que cuando los turistas mantienen el contacto social con sus familias y amigos durante sus viajes, los tiempos y las distancias dadas por los espacios geográficos "se encogen” (Moores, 2005), y esta comunicación de cierta forma puede desencadenar una nueva reflexión de la experiencia del viaje provocada por la retroalimentación inmediata que recibe el viajero.

Goossens (2000) ha señalado cómo los sentimientos y emociones son una parte esencial de la experiencia turística y responden a situaciones llenas de significado para el turista, que pueden ocurrir antes, durante o después del viaje. Munar y Jacobsen (2014) han estudiado las motivaciones para compartir en medios de comunicación social en línea, indicando que las redes sociales no solo permiten a los turistas compartir información, sino también experiencias. Según los autores, las experiencias son personales, y constituyen la percepción y la reacción del individuo con respecto a lugares, espacios y servicios turísticos que incluyen tanto la información de facto acerca de los mismos, como las emociones, imaginaciones y fantasías relacionadas con el viaje. Los autores 
señalan la forma en que, al momento de compartir en línea, estos sentimientos y emociones se observan por ejemplo a través de las fotografías, y se refuerzan y reviven mediante los comentarios y emoticonos que las acompañan.

En resumen, estudios previos muestran cómo el smartphone puede modificar la experiencia de viaje. Esa herramienta acompaña al viajero en todo momento, brindándole información en tiempo real, cuando la necesita, otorgándole facilidad de movimiento, así como posibilidades de improvisación. Más allá de la información, el smartphone cambia la experiencia de viaje cuando los viajeros, aun lejos de su lugar de origen, se sienten conectados, compartiendo su experiencia con fotos, videos, o simplemente cambiando su ubicación actual en las redes sociales. Estas experiencias compartidas están cargadas de significado, emociones y sentimientos, tanto al subirlas a la red como en la retroalimentación inmediata que se recibe mediante comentarios, likes y emoticonos. Desde este punto de vista, el teléfono inteligente se convierte en un intermediario entre el viajero y el destino: no solo le permite acceder a él, sino también apropiarse del mismo a través de las múltiples herramientas y aplicaciones de viaje disponibles.

\section{El teléfono inteligente y las aplicaciones como herramientas de viaje}

Los viajeros usualmente necesitan acceso a gran cantidad de información que les ayude a comprender su entorno, para tomar decisiones durante el viaje (Gretzel et al., 2006). Desde ese punto de vista, con su capacidad de portabilidad y rápida conexión a internet, los teléfonos inteligentes parecen satisfacer a la perfección los requerimientos de los viajeros (Buhalis y Law, 2008). Estas características le brindan al smartphone la capacidad para convertirse en una herramienta útil de información de viajes y un portal para acceder a diferentes facilidades turísticas.

De acuerdo con Euromonitor International, en el año 2013, casi 50 \% de los usuarios europeos de un smartphone lo utilizan para hacer reservaciones de todo tipo durante sus vacaciones. Adicionalmente se presentan cifras que contribuyen a caracterizar el uso del smartphone por estadounidenses: $85 \%$ de los viajeros utiliza su smartphone en el extranjero; $30 \%$ ha usado aplicaciones para 
(Des)conexión durante el viaje turístico: uso de smartphones por parte de viajeros independientes en la ciudad de Guayaquil

obtener ofertas en hoteles; $72 \%$ ha publicado alguna foto de sus vacaciones en alguna red social; 46 \% comparte su ubicación durante sus vacaciones por medio de alguna red social; 70 \% actualiza su estado de Facebook mientras está de vacaciones. Además se indica que los cinco principales usos del smartphone durante los viajes son tomar fotos, consultar mapas, buscar restaurantes, buscar actividades y atracciones locales, y consultar los estados de vuelos (Euromonitor, 2013). En paralelo, “Our Mobile Planet”, un estudio realizado por la compañía Google (Tns/Google, 2012), presenta datos estadísticos sobre acciones realizadas luego de consultar información local mediante teléfonos inteligentes, es decir, cómo los viajeros actúan de acuerdo con lo que exploran en sus móviles. Revisan información local, $92 \%$ de los viajeros de Estados Unidos, $84 \%$ de Inglaterra y $88 \%$ de España, y, de estos, $89 \%$ de estadounidenses, $80 \%$ de ingleses y $80 \%$ de españoles realizan algún tipo de acción luego de consultar la información local. Esto convierte al smartphone en una herramienta de toma de decisiones durante los viajes.

El uso de teléfonos inteligentes en los viajes se facilita a través de las apps o aplicaciones móviles, que son programas informáticos diseñados para ser ejecutados en teléfonos inteligentes, tabletas u otros dispositivos móviles. Las dos tiendas virtuales de aplicaciones móviles: Google Play y Apple App Store albergan unas 800000 aplicaciones cada una; se calcula que un billón doscientas mil personas usaban aplicaciones móviles en el 2012, y que este número podría llegar a 4.4 billones de usuarios en 2017 (mobiThinking, 2014). Según el estudio de TripAdvisor, la cifra de reservas de viajes hechas mediante aplicaciones móviles se duplicó de 2014 a 2015, y llegó a 8 \% de las reservas realizadas por los encuestados (TripAdvisor, 2015). En consecuencia, las aplicaciones móviles para viajes y turismo no solo están aumentando exponencialmente, sino que están cambiando la forma en que el turista busca información, se comporta, consume y toma decisiones (Kramer, Modsching, Hagen y Gretzel, 2007; Wang et al., 2011; Dickinson et al., 2014).

Wang et al. (2011), analizan las aplicaciones de la plataforma de Apple, y las categorizan por el tipo de información o utilidad que proveen a los viajeros. En los resultados de su estudio clasifican las aplicaciones en: guías turísticas (Vegas Mate, NYC way), agencias de viaje en línea (Travelocity, hotel search), guías de atracciones (Disney World Dining), guías de entretenimiento (Talk 
radio, Trip Journal), transportes locales (Metro Paris, Subway), localización de restaurantes (Happy Hours, Urbanspoon), entre otras. A su vez, Dickinson et al. (2014) destacan que las aplicaciones móviles son desarrolladas no solo por prestadores de servicios turísticos y empresas privadas, sino también por organizaciones de gestión de destinos, gobiernos nacionales y locales. Según los autores, los teléfonos inteligentes y las aplicaciones móviles hacen que el viajero pueda alinear sus necesidades, preferencias, recursos y restricciones de tiempo, con la oferta de servicios y actividades en el lugar de destino, lo que le brinda más libertad que nunca a la hora de movilizarse. Analizar el uso de los teléfonos inteligentes, patrones de desplazamiento, así como la toma de decisiones por parte de los turistas permitiría, por ejemplo, mejorar la información y la oferta de productos y destinos turísticos.

\section{Metodología}

Con el fin de obtener información a profundidad sobre cómo los viajeros independientes utilizan su teléfono inteligente en la ciudad de Guayaquil, se realizó un estudio cualitativo. Una de las principales características de la investigación cualitativa es el naturalismo, según el cual la realidad social se entiende como "realmente es", y se obtienen amplias descripciones de las opiniones e interacciones de las personas (Gubrium y Holstein, 1997). La investigación cualitativa recoge las interpretaciones subjetivas y opiniones de los participantes, y da como resultado vastas y completas descripciones de las percepciones individuales de cada sujeto (Wang et al., 2013).

\section{Diseño de instrumentos y recolección de datos}

El muestreo de la investigación cualitativa requiere establecer criterios de selección de informantes que se conecten a las preguntas y objetivos de investigación (Miles, Huberman y Saldaña, 2014: 46). Para seleccionar a los informantes se consideraron tres criterios: poseer un smartphone desde al menos un año antes; haber visitado la ciudad de Guayaquil al menos una vez durante el último año, por motivos de ocio, y haber culminado su visita a la ciudad de Guayaquil al momento de la entrevista. Además, se eligieron visitantes que hubieran pernoctado en la ciudad al menos una noche, para que el informante 
(Des)conexión durante el viaje turístico: uso de smartphones por parte de viajeros independientes en la ciudad de Guayaquil

hubiera tenido mayor tiempo de uso de su dispositivo móvil en el destino. Para recolectar la información se condujeron ocho entrevistas semiestructuradas. Los informantes fueron localizados mediante anfitriones que hospedaban a turistas en Guayaquil y fueron entrevistados por Skype o de manera presencial, una vez que culminaron su experiencia de viaje. Se realizaron entrevistas en línea, puesto que algunos informantes ya se encontraban en su país de origen.

El proceso de la recolección de datos abarcó tres etapas principales: la primera consistió en elaborar la herramienta para recolectar información. Para plantear las preguntas se revisó la literatura, es decir, los métodos utilizados y los resultados obtenidos en estudios previos. Las preguntas responden además a la realidad local, así como a los objetivos de la investigación, que eran conocer el uso del smartphone por parte de los turistas de la ciudad de Guayaquil, por una parte, y analizar cómo este uso influye en la experiencia de viaje, por otra. En consecuencia, en el cuestionario se enfatizaron preguntas orientadas a conocer el uso del dispositivo durante el viaje (Wang et al., 2013), y no antes o después del mismo (Jennings y Weiler, 2006; Gretzel et al., 2006) por considerarse que el estudio debía enfocarse en cómo los viajeros utilizan su teléfono inteligente en el destino. Adicionalmente se realizaron preguntas para obtener información general del viaje realizado (véase cuadro 1).

La segunda etapa consistió en aplicar las entrevistas piloto. Se realizaron tres entrevistas piloto que sirvieron para verificar la cobertura de los temas y la comprensión del cuestionario por parte de los participantes (Robson y McCartan, 2016). Los datos obtenidos en estas entrevistas fueron parte del análisis de resultados. Esas tres primeras entrevistas sugirieron modificar algunas preguntas con la finalidad de hacerlas más claras para el informante y a la vez, mejorar la calidad de las respuestas.

En la tercera etapa se aplicó el cuestionario, mediante entrevistas semiestructuradas con los informantes. En total se llevaron a cabo ocho entrevistas; el número de informantes fue determinado por el nivel de saturación de datos, es decir, se hicieron entrevistas hasta que no se obtuvieron nuevos datos. La saturación puede ser de varios tipos: Glaser y Strauss (1967, p. 65) definen la saturación de datos como el punto en el cual el investigador no encuentra datos adicionales, es decir, cuando halla datos similares una y otra vez. En este método el investigador busca variaciones extremas del concepto hasta el 
agotamiento. La saturación ocurre cuando todas las principales variaciones del fenómeno han sido identificadas e incorporadas al conocimiento emergente (Guest, Bunce y Johnson, 2006).

\section{Análisis de la información}

Como parte del análisis, se codificaron las entrevistas realizadas durante el proceso de recolección de información (Miles et al., 2014). Charmaz (2001) describe la codificación como la etapa entre la recolección de datos y la explicación del significado, en la que un código es una construcción investigador-entrevistado que simboliza los atributos interpretados de las respuestas de cada individuo para, finalmente, identificar patrones, construir teorías, y otros procesos analíticos.

En su etapa incial, la codificación reveló 24 códigos divididos en cinco categorías principales: uso, aplicaciones, etapas de viaje, experiencia de viaje y características del informante. Luego se revisaron los códigos y la información que contenía cada uno, es decir, se inició un proceso de evaluación de códigos con base en los objetivos de la investigación, e información relevante para el estudio. Se procedió a descartar y unificar varios códigos (Miles et al., 2014). El descarte se dio por dos motivos: 1) la información obtenida no respondía a los objetivos de la investigación o no era relevante para el estudio, y 2) la información del código era insuficiente, es decir, no tenía mayor cantidad de datos o descripciones. Se agruparon por códigos similares, o porque varios códigos respondían al mismo objetivo de la investigación. Luego del proceso, se obtuvieron siete códigos agrupados en dos grandes categorías de acuerdo con los objetivos de la investigación: uso en el destino y el aporte en la experiencia de viaje (véase cuadro 1).

Cabe destacar que, al final del proceso de recolección de datos, además del perfil previamente establecido para la investigación (criterios para la selección de informantes), los participantes mostraron características adicionales que pueden influir los datos de alguna manera: todos son latinos (Argentina,

CUADRo 1. Relación entre las categorías d emergentes durante0a3 proces 
(Des)conexión durante el viaje turístico: uso de smartphones por parte de viajeros independientes en la ciudad de Guayaquil

CuAdro 1. Relación entre las categorías de análisis, preguntas del cuestionario y temas emergentes durante el proceso de codificación e interpretación

(finaliza)

\begin{tabular}{|c|c|c|}
\hline $\begin{array}{l}\text { Categorías DE } \\
\text { ANÁLISIS }\end{array}$ & $\begin{array}{l}\text { CUESTIONARIO DE PREGUNTAS } \\
\text { SEMIESTRUCTURADAS }\end{array}$ & TEMAS EMERGENTES \\
\hline $\begin{array}{l}\text { Información general } \\
\text { del viaje }\end{array}$ & $\begin{array}{l}\text { País de residencia } \\
\text { Hace cuánto tiempo posee un } \\
\text { smartphone } \\
\text { Fecha de su más reciente viaje a } \\
\text { Guayaquil } \\
\text { Días de estadía } \\
\text { Actividades realizadas en Guayaquil }\end{array}$ & $\begin{array}{l}\text { País de residencia } \\
\text { Tiempo que tiene un } \\
\text { smartphone } \\
\text { Lugares de visita } \\
\text { Permanencia en el destino }\end{array}$ \\
\hline $\begin{array}{l}\text { Uso del smartphone } \\
\text { durante el viaje }\end{array}$ & $\begin{array}{l}\text { Uso del smartphone durante la visita } \\
\text { a Guayaquil } \\
\text { Aplicaciones más usadas durante la } \\
\text { visita a Guayaquil } \\
\text { Portales de búsqueda más usados } \\
\text { durante la visita a Guayaquil } \\
\text { Uso y descarga de aplicaciones de } \\
\text { turismo del destino } \\
\text { Aplicaciones que más utiliza en su } \\
\text { vida cotidiana } \\
\text { Uso del smartphone para planificar } \\
\text { el viaje a Guayaquil } \\
\text { Uso del smartphone después de la } \\
\text { visita a Guayaquil }\end{array}$ & $\begin{array}{l}\text { Tiempo de uso } \\
\text { Problemas de uso } \\
\text { Finalidad de uso } \\
\text { Lugares de uso } \\
\text { Momento de uso } \\
\text { Necesidad/Motivación de } \\
\text { uso } \\
\text { Problemas de uso } \\
\text { Portales/aplicaciones más } \\
\text { utilizados durante el viaje } \\
\text { Portales/aplicaciones más } \\
\text { utilizados durante la vida } \\
\text { cotidiana } \\
\text { Portales/aplicaciones más } \\
\text { utilizados antes o después } \\
\text { del viaje }\end{array}$ \\
\hline $\begin{array}{l}\text { Aporte a la } \\
\text { experiencia de viaje }\end{array}$ & $\begin{array}{l}\text { Ventajas del uso del smartphone } \\
\text { durante los viajes turísticos } \\
\text { Sentimientos relacionados con estar } \\
\text { conectado a internet durante el viaje } \\
\text { Sentimientos relacionados con usar } \\
\text { el smartphone durante el viaje } \\
\text { Diferencias entre viajar con o sin } \\
\text { smartphone }\end{array}$ & $\begin{array}{l}\text { Aporte a la experiencia de } \\
\text { viaje } \\
\text { Sentimientos de conexión y } \\
\text { desconexión con el lugar de } \\
\text { origen/ destino }\end{array}$ \\
\hline
\end{tabular}

Fuente: Elaboración propia. 
Perú, Chile, Colombia); su edad fluctúa entre 22 y 33 años, y todos son viajeros independientes, es decir, no llegaron a la ciudad con un paquete turístico organizado. Esta última característica se considera de especial relevancia para los resultados de la investigación, ya que es posible que quienes viajan con un paquete organizado tengan diferentes necesidades de información.

\section{Caracterización de uso del smartphone en Guayaquil}

El análisis de las entrevistas reveló cómo los visitantes hacen uso de su smartphone durante su visita a la ciudad de Guayaquil. Se identificaron estos temas principales: objetivo o motivación de uso, aplicaciones y portales de búsqueda más utilizados, y problemas de uso.

\section{Objetivos de uso}

Los principales usos del smartphone en el destino según las entrevistas realizadas son: comunicación (7 de 8 informantes), uso de mapas (6 de 8 informantes), búsqueda de información de la ciudad (5 de 8 informantes), fotografía (3 de 8 informantes), música y otros (2 de 8 informantes), búsqueda de alojamiento (1 de 8 informantes) (figura 1).

La comunicación es de gran relevancia para los turistas durante sus viajes. Por ejemplo, varios informantes relataron que se comunicaban con sus familiares al llegar a su destino; algunos se comunicaban además con sus amigos o conocidos para compartir información sobre su viaje, su nueva ubicación.

Sobre todo para comunicarme, la de WhatsApp con mi familia y mis amigos allá en Argentina; cuando localizamos algún hospedaje o algún lugar donde quedarnos [informante 3].

Además, mediante el smartphone los turistas logran contactarse con conocidos de la ciudad, algunos participantes indicaron, por ejemplo, que al llegar a Guayaquil se contactaban con amigos de la ciudad para encontrarlos o realizar alguna actividad en la ciudad.

De hecho cuando me contacté con una amiga para ir a la Isla Santay y buscar información con el smartphone [informante 8]. 
(Des)conexión durante el viaje turístico: uso de smartphones por parte de viajeros independientes en la ciudad de Guayaquil

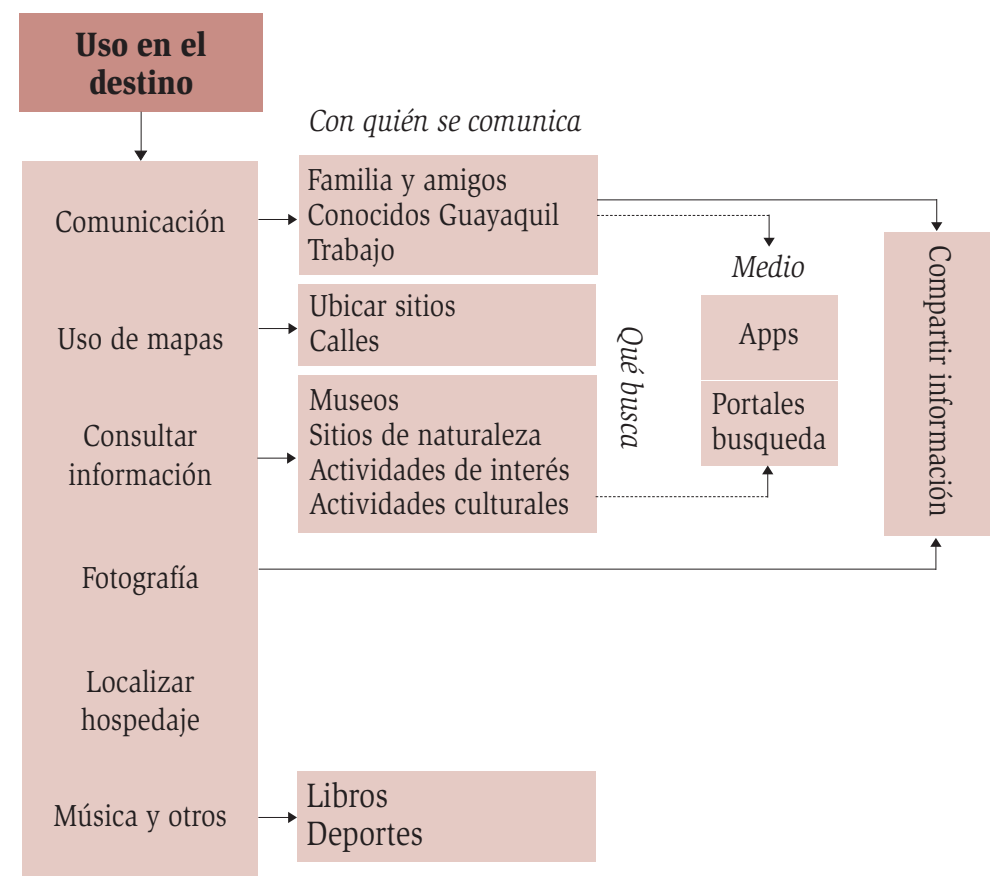

Fuente. Elaboración propia.

Figura 1. Uso del teléfono inteligente en el destino

Buscar información del sitio que se visita es un ejercicio básico de los turistas. En efecto, los informantes indicaron que los temas más buscados cuando estuvieron en Guayaquil fueron lugares de interés, actividades para realizar, museos, sitios de naturaleza, actividades culturales.

Y después lugares para visitar, desde los horarios de los museos, dónde tomar el bus o los lugares que queríamos visitar de naturaleza, y los horarios para visitarlos; comentarios de otras personas que hayan recorrido esos lugares [informante 3].

El uso de los mapas y el GPS mediante el smartphone es muy popular según los informantes, quienes reportan utilizar el GPs y mapas en todo momento en el destino para ubicar sitios de interés, calles, etcétera. 
Sí, [...] para ver el mapa de la ciudad, todo el tiempo estaba mirándolo, pero cuando tenía wifi, porque no tenía datos en Ecuador, entonces [...] cuando tenía wifi lo usaba para ver el mapa y ubicarme en las calles [informante 5].

\section{Aplicaciones y portales de búsqueda más utilizados}

En las entrevistas se solicitó a los informantes nombrar las aplicaciones que más usan durante sus viajes en relación con las más usadas en su vida cotidiana, con el fin de contrastar si se modifica el patrón de uso de aplicaciones móviles durante los viajes.

Las tres aplicaciones más usadas durante los viajes son: mapas y GPS (8 de 8 informantes), WhatsApp (7 de 8 informantes), Facebook (7 de 8). Otras aplicaciones usadas en los viajes son el correo electrónico (3 de 8), TripAdvisor (1 de 8), CouchSurfing (1 de 8). Por otra parte, las tres aplicaciones que los informantes reportan usar principalmente en su vida cotidiana y fuera de la experiencia de viaje, son: WhatsApp (8 informantes), Facebook (8 informantes) y correo electrónico (2 de 8). Otras aplicaciones nombradas fueron Skype (1 de 8), Line (1 de 8), Twitter (1 de 8), aplicaciones de taxi (1 de 8), aplicaciones de transporte público (1 de 8).

En general, según los informantes, el patrón de uso de las aplicaciones se conserva tanto durante el viaje como en la vida cotidiana (figura 2). Las aplicaciones que mantienen su patrón de uso son especialmente de comunicación y redes sociales, sin embargo, sí se evidencia que tienden a revisar más las aplicaciones de mapas durante su viaje que en su vida cotidiana.

Además, el informante 5 señaló la necesidad de que la ciudad de Guayaquil cuente con una aplicación que brinde información sobre el transporte público urbano.

Porque muchas ciudades tienen sus rutas de metro, o del [transporte] que tienen aquí en Guayaquil, metrovía de buses, en una aplicación, entonces uno puede buscar las rutas y frecuencias [...], pero aquí en Guayaquil no encontré una aplicación que hiciera eso [informante 5]. 
(Des)conexión durante el viaje turístico: uso de smartphones por parte de viajeros independientes en la ciudad de Guayaquil
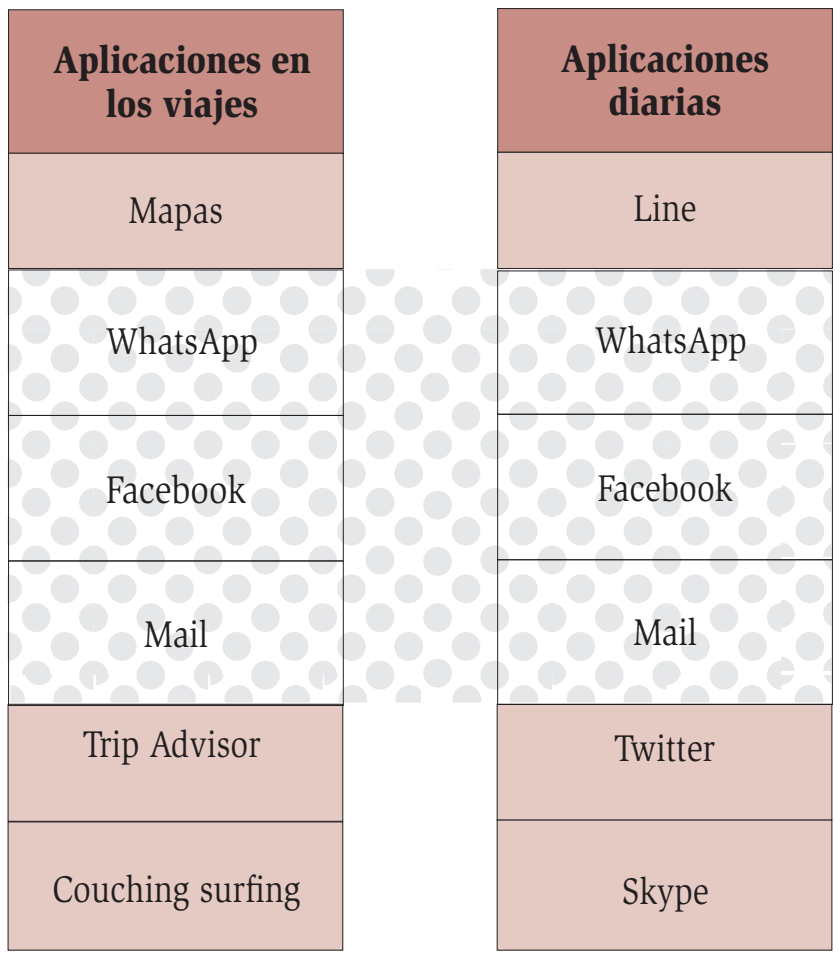

Fuente: Elaboración propia.

Figura 2. Patrón de uso de aplicaciones durante el viaje vs. la vida cotidiana

Con respecto a los portales de búsqueda, los más consultados por los viajeros son: Google (todos los informantes), comentarios en blogs o Facebook (2 de 8), agendas culturales de la municipalidad (1 de 8), CouchSurfing (1 de 8), Hostel World (1 de 8), TripAdvisor (1 de 8) (figura 3). 


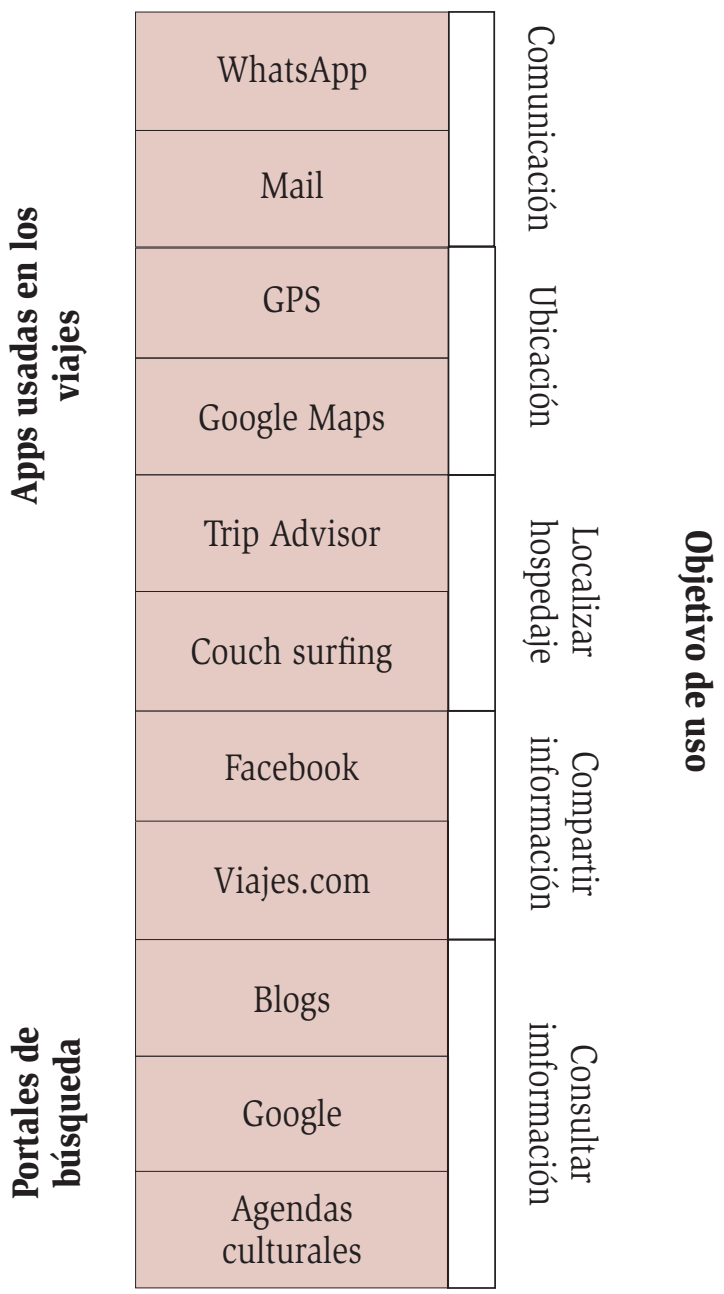

Fuente: Elaboración propia.

FiguRa 3. Aplicaciones más usadas durante los viajes 
(Des)conexión durante el viaje turístico: uso de smartphones por parte de viajeros independientes en la ciudad de Guayaquil

\section{Problemas de uso}

Al momento de visitar la ciudad, la mayoría de nuestros informantes no tenía datos de navegación en su teléfono inteligente, por lo cual debían conectarse a un punto público de wifi para navegar, o utilizar alguna aplicación en su móvil. Los informantes se quejaron además de que hay lugares públicos donde se anuncia una conexión gratuita a internet a la que no pudieron conectarse.

Pues cuando tenía wifi, porque no tenía datos en Ecuador, entonces cuando tenía wifi lo usaba para ver el mapa y ubicarme en las calles [informante 5].

Por ejemplo, tenía páginas que busqué sobre qué lugares visitar, las tenía guardadas en el celular para [cuando disponía de wifi], poder también buscar [informante 7].

En la ciudad hay muchos lugares que dicen que tienen wifi, pero no engancha, como por ejemplo en las metrovías o en el Malecón [...] también hay zonas libres de wifi, donde podría tranquilamente consultar algo; pero no [informante 1].

\section{Uso del smartphone y su relación con la experiencia de viaje}

Dentro de la categoría "experiencia de viaje" obtuvimos tres temas que muestran la influencia del smartphone en lo que el visitante percibe como su propia experiencia al viajar: aporte en la experiencia de viaje, sentimientos generados y modificación de la interacción con el destino.

\section{Aporte en la experiencia de viaje}

Las facilidades que otorga el uso del smartphone al momento de viajar son muy diversas: todos los entrevistados mencionaron al menos una manera en la que el smartphone aporta algo a la experiencia de viaje. Los informantes 2, 3, 4 y 5, por ejemplo, indicaron que la mayor contribución del smartphone en sus viajes es el uso de mapas, porque les dio facilidad de movimiento. La agilidad para obtener información cuando la necesitan y la oportunidad de optimizar su tiempo en el destino son otras ventajas que algunos de los entrevistados encontraron en el teléfono inteligente (informantes 1, 7 y 8).

Para mí lo mejor es la cuestión de los mapas, [...] porque en otros viajes me ha pasado que he llegado y los puntos turísticos están cerrados, entonces por ahí (por el 
smartphone) tengo acceso a un mapa y [puedo] averiguar acerca de un alojamiento y acceder a informaciones concretas [informante 2].

Se hace más eficiente el viaje; no tienes que buscar una dirección tres veces, no tienes que preguntarle a la gente; por ahí lo buscas y ahí llegas [informante 4].

Yo había ido a Ecuador antes y de hecho es mucho más cómodo, porque antes para revisar cada cosa tenía que ir a un cibercafé, conseguir una notebook; era más pérdida de tiempo [...] el tiempo de buscar que uno tenía era más limitado. Con el smartphone es todo instantáneo: como lo traigo conmigo puedo revisar cualquier información, hay un cambio gigantesco a estar con y sin el smartphone [informante 8].

La capacidad de comunicarse y compartir información es, para algunos entrevistados, un gran aporte; tener la rapidez para compartir información, relatar sus sensaciones y vivencias al instante, es lo que mejora su experiencia (informantes 1,5 y 8 ).

No podías comunicarte con la gente que está pendiente de ti [informante 5].

Ahora con el smartphone casi es una necesidad ir contando todo lo que uno va haciendo. [...] antes uno tenía que esperar conectarse a una computadora para contar o narrar algo, y a veces incluso daba flojera hacerlo; pero ahora, de forma instantánea, tomas la foto y la envías. Ha cambiado mucho [informante 8].

Se solicitó a los entrevistados justificar por qué preferían usar el smartphone sobre otras opciones. Las respuestas incluyeron: portabilidad (5 de 8 informantes), comodidad (3 de 8), rapidez y exactitud (3 de 8 ), versatilidad de uso (3 de 8), facilidad de acceso a información (2 de 8).

Todo está en el smartphone; la cantidad gigante de aplicaciones que tiene es supercómoda; es muy rápido, exacto, incluso, versus una computadora, el smartphone es portátil, cabe en tu bolsillo. Es la diferencia [informante 8].

Cuando decidí viajar, yo reduje todo ahí, para bajar libros, escuchar música [informante 3].

Es más fácil de usar, más pequeño, más cómodo [...] y no solo eso, sino que todo es accesible en cualquier momento [informante 4]. 
(Des)conexión durante el viaje turístico: uso de smartphones por parte de viajeros independientes en la ciudad de Guayaquil

\section{Sentimientos generados}

Un tema que los informantes mencionaron frecuentemente durante las entrevistas son los sentimientos que se generaban en ellos al usar su dispositivo móvil durante sus viajes. Según sus testimonios, de cierto modo el teléfono inteligente deja de ser solo una ayuda logística, y su uso otorga además satisfacción emocional. La generación de estos sentimientos es una de las claves de la influencia del smartphone en la experiencia de viaje. Los principales sentimientos identificados son: seguridad (2 de 8 informantes), ayuda y comodidad (2 de 8), libertad de movimiento (1 de 8), poder de decisión (1 de 8), placer (1 de 8).

Lo podría decir en una palabra, y es placer; se podría decir que es más [...] placer y sentirme siempre conectado [...] es placentero compartir de forma instantánea lo bien que lo está pasando uno [informante 8].

Yo creo que me da seguridad en el sentido de que... sé dónde estoy yendo, sé los lugares; me gusta estar ubicado [informante 5].

Como sentir que es tu gPs para moverte con libertad [informante 3].

\section{(Des)conexión con el lugar de origen}

Una parte fundamental del uso del smartphone es la capacidad que otorga a los viajeros de compartir información y sus experiencias al instante. Los turistas comparten sus experiencias en el destino enviando o publicando mensajes de texto, fotos y videos, actualizando sus estados en las redes o checking-in, es decir, registrando en las redes sociales su ingreso o llegada a lugares como hoteles, aeropuertos 0 atracciones turísticas.

Los resultados nos muestran que viajeros se encuentran constantemente conectados con su lugar de origen, enviando fotos y videos. Además, algunos comparten sus vivencias, comentarios u opiniones del destino visitado mediante blogs de viajero o páginas de viajes.

Sí lo utilizo, porque estoy en una página que se llama viajeros.com en la cual uno narra de fondo la vivencia que tuvo en otro lugar, entonces [...] la verdad como yo casi no utilizo notebook, y siempre ando con el smartphone, lo uso para escribir mi experiencia en esa página [informante 8]. 
Ahora estaba subiendo fotos de los lugares que recorrí, pero en las redes sociales y porque no tengo blog de viajero [informante 3].

\section{(Des)conexión con el destino}

En esta sección se analizaron las respuestas de los informantes que revelaban cierta dependencia respecto del uso del teléfono inteligente, y cómo este puede interrumpir la interacción entre el destino y el visitante.

De alguna manera hay cierta dependencia, $[\ldots]$ porque cuando uno se conecta a internet la comunicación con lo que uno está viviendo se acaba y pasa a ser con el elemento tecnológico [informante 2].

Sí modifica la interacción [social] a veces, porque si vos querés no necesitas comunicarte con nadie más [en el destino] que con el teléfono para moverte; eso es lo que creo que se modifica [informante 3].

Bueno, de hecho, a veces estás muy pegado al celular y te puedes desconectar de lo que estás viendo, del paisaje, pero trato de evitarlo; si estoy en un sitio que es de naturaleza trato de estar desconectado y no tener mucho el celular [informante 7].

\section{Discusión y conclusiones}

Los resultados obtenidos en la investigación revelan cómo los viajeros independientes hacen uso de sus dispositivos móviles en la ciudad de Guayaquil. Según el análisis, el uso principal es la comunicación, sobre todo con amigos y familiares en el lugar de origen, seguido de la consulta de mapas, la consulta de información turística en general, y la toma de fotografías. Si bien es verdad que los resultados de uso del dispositivo de cierta forma coinciden con los estudios de Euromonitor (2013) y TripAdvisor (2015), en los cuales los principales usos tienen que ver con la búsqueda en mapas, de información turística y toma de fotografías, el tema de la comunicación y la posibilidad de compartir información inmediata con amigos y familiares no solo resulta emergente, sino que tiene una relación importante con la experiencia turística del visitante.

Respecto a las aplicaciones móviles, las de mapas suelen ser las más usadas (Google Maps), ya que los turistas recurren a ellas para ubicar los lugares turísticos de manera inmediata y en tiempo real. Otras aplicaciones utilizadas 
(Des)conexión durante el viaje turístico: uso de smartphones por parte de viajeros independientes en la ciudad de Guayaquil

con frecuencia incluyen servicios de mensajería instantánea (WhatsApp), redes sociales (Facebook) y correo electrónico. Se evidenció, además, que durante los viajes no se modifica el patrón de empleo de las aplicaciones más usadas en la vida cotidiana, sin embargo, se incorporan nuevas como TripAdvisor o CouchSurfing.

La investigación buscó, por otra parte, identificar si el uso del teléfono inteligente altera la experiencia de viaje: si hay sentimientos y emociones relacionados con el uso del dispositivo. Los resultados indican que las peculiaridades del dispositivo dan comodidad y flexibilidad a los viajeros, ofreciéndoles soluciones e información en tiempo real cuando lo requieran. Los turistas se sienten empoderados cuando utilizan un teléfono inteligente, ya que la información disponible en internet amplía su poder de decisión y permite la improvisación y la libertad de movimiento. Gretzel et al. (2006) explican que el internet provee al viajero de información y opciones sin precedentes, lo que le permite controlar como nunca antes los procesos de consumo de la experiencia turística. Los sentimientos de poder, control y libertad que los participantes describen en sus testimonios son aspectos que podrían profundizarse en futuras investigaciones.

A pesar de que el uso del teléfono inteligente influye de manera positiva en la experiencia de viaje, los participantes también mencionaron formas en que el dispositivo puede interrumpir la conexión con el destino al generar sentimientos de dependencia y debido a la necesidad de estar conectado constantemente. Asimismo, el uso de aplicaciones de mapas y otras fuentes de búsqueda de información en línea reduce la necesidad de interacción de los visitantes con los residentes de la ciudad o incluso con otros viajeros, limitando la posibilidad de nuevas experiencias basadas en la interacción social en el destino (Carmichael, 2005). Un participante hizo énfasis en cómo "la comunicación con lo que se está viviendo se acaba” para enfocarse en la comunicación con el dispositivo. La idea de decapsulación (Jansson, 2007; Wang y Fesenmaier, 2013) explica con claridad este sentimiento en el que el uso del teléfono móvil rompe con la distinción entre origen y destino o la distinción entre tiempo de trabajo y de ocio, permitiendo que el viajero esté presente en varios lugares o haciendo varias cosas al mismo tiempo.

En resumen, los resultados indican que el smartphone es principalmente un canal de comunicación entre los turistas y las personas en su entorno habitual 
de residencia y, por otra parte, que la conexión con el destino, así como la interacción con los habitantes de ese destino puede verse reducida por el uso del teléfono inteligente. De esta forma, aun en la distancia, el smartphone evita la ruptura de la comunicación con amigos y familiares en casa, encogiendo los espacios geográficos (Wang et al., 2013), facilitando la conexión con el lugar de origen y dificultando la desconexión del turista con respecto a su vida cotidiana. Estos sentimientos de conexión con el origen, (des)conexión con el destino pueden llevarnos a reconsiderar el significado mismo de la experiencia turística. En efecto, las propias definiciones de turismo han mantenido de manera tradicional a la actividad separada de lo que sucede en la vida cotidiana (Larsen, 2008; McCabe, 2002). No obstante, es evidente que el uso de los dispositivos móviles puede generar alteraciones a la idea tradicional de "escape", al permitir la conexión permanente con ese lugar de origen, e impedir la separación ontológica del turista con su vida cotidiana y con su lugar habitual de residencia.

Por último, con respecto a restricciones, los participantes mencionaron la necesidad de que la ciudad cuente con una aplicación móvil para consultar rutas y horarios de transporte público urbano. Además varios informantes dijeron que una de las restricciones más grandes para el uso del teléfono inteligente una vez en Guayaquil era el acceso a redes públicas de wifi. Los testimonios de los participantes pueden ser tenidos en cuenta a la hora de planificar la presencia digital de destinos turísticos, en especial en el contexto de proyectos relacionados con ciudades digitales o inteligentes.

La presente investigación identificó cualitativamente y de manera exploratoria los usos del teléfono inteligente por parte de viajeros independientes y su relación con la experiencia de viaje en el destino Guayaquil. Futuras investigaciones podrían mantenerse en un enfoque cualitativo para profundizar en temas puntuales, como los sentimientos de conexión con el lugar de origen/ desconexión con el destino, así como las sensaciones de poder y libertad que experimentan los viajeros. Los resultados obtenidos también podrían servir de base para diseñar un instrumento de medición que abarque una muestra más amplia de participantes. 
(Des)conexión durante el viaje turístico: uso de smartphones por parte de viajeros independientes en la ciudad de Guayaquil

\section{Fuentes consultadas}

Aurigi, A. (2005). Making the Digital City: The Early Shaping of Urban Internet Space. Burlington: Ashgate.

Benítez Victoria, V. y Bermúdez Moreno, J. D. (2015). Evaluación de la viabilidad del desarrollo de una app especializada en turismo de naturaleza para la ciudad de Pereira (Tesis de grado). Universidad Tecnológica de Pereira, Pereira.

Buhalis, D. y Law, R. (2008). Progress in information technology and tourism management: 20 years on and 10 years after the Internet-The state of eTourism research. Tourism Management, 29(4), 609-623.

Carmichael, B. (2005). Understanding the wine tourism experience for winery visitors in the Niagara region, Ontario, Canada. Tourism Geographies, $7(2), 185-204$.

Charmaz, K. (2001). Grounded theory: methodology and theory construction. En International Encyclopedia of the Social and Behavioral Sciences (pp. 6396-6399). Oxford: Elsevier.

Cuadro Guerrero, S. L. y Ruiz Vergara, W. O. (2017). Diseño de una herramienta tecnológica que facilite la identificación de atractivos turísticos alrededor a las principales paradas de la metrovia (Tesis de grado). Escuela Superior Politécnica del Litoral, Guayaquil.

Dickinson, J. E., Ghali, K., Cherrett, T., Speed, C., Davies, N. y Norgate, S. (2014). Tourism and the smartphone app: Capabilities, emerging practice and scope in the travel domain. Current Issues in Tourism, 17(1), 84-101.

Euromonitor International. (2013). World Travel Market Industry Report and Global Trends Report. Londres: World Travel Market.

El Universo. (25 de julio de 2015). Jaime Nebot: Guayaquil será pronto la primera ciudad digital de Sudamérica. El Universo.

El Universo. (3 de agosto de 2015). 4.000 bachilleres reciben tablets ofrecidas por el Municipio de Guayaquil. El Universo.

Florido-Benítez, L. (diciembre, 2016). Análisis de las fortalezas y debilidades del mobile marketing como herramienta de promoción de los destinos turísticos. Revista Turydes: Turismo y Desarrollo, 21. 
GAD Municipal de Guayaquil. (2014). Guayaquil ciudad digital. Alcaldía de Guayaquil. Recuperado de http://www.guayaquil.gob.ec/guayaquil-digital [2017, 19 de abril].

Gretzel, U., Fesenmaier, D. R. y O'Leary, J. T. (2006). The transformation of consumer behaviour. En D. Buhalis y C. Costa (eds.), Tourism Business Frontiers: Consumers, Products and Industry (pp. 9-18). Oxford: Elsevier.

Glaser, B. y Strauss, A. (1967). The Discovery Grounded Theory: Strategies for Qualitative Inquiry. Chicago: Aldin.

Goossens, C. (2000). Tourism information and pleasure motivation. Annals of Tourism Research, 27(2), 301-321.

Gubrium, J. F. y Holstein, J. A. (1997). The New Language of Qualitative Method. Oxford: Oxford University Press on Demand.

Guest, G., Bunce, A. y Johnson, L. (2006). How many interviews are enough? An experiment with data saturation and variability. Field Methods, 18(1), 59-82.

INEc. (2016). En cinco años se quintuplicaron los usuarios de teléfonos inteligentes. Recuperado de http://www.ecuadorencifras.gob.ec/en-cincoanos-se-quintuplicaron-los-usuarios-de-telefonos-inteligentes/ [2017, 19 de abril].

Jansson, A. (2007). A sense of tourism: New media and the dialectic of encapsulation/decapsulation. Tourist Studies, 7(1), 5-24.

Jennings G. R. y Weiler, B. (2006). Mediating meaning: Perspective on brokering quality tourism experiences. En G. Jennings y N. Polovitz (eds.), Quality Tourism Experiences (pp. 57-58). Burlington: Elsevier.

Kramer, R., Modsching, M., Hagen, K. y Gretzel, U. (2007). Behavioural impacts of mobile tour guides. En M. Sigala, L. Mich y J. Murphy (eds.), Information and Communication Technologies in Tourism 2007 (pp. 109118). Viena: Springer.

Larsen, J. (2008). De exoticizing tourist travel: Everyday life and sociality on the move. Leisure Studies, 27(1), 21-34.

Mariño Arias, L. V. (2012). Guía turística interactiva para teléfonos móviles, para la ciudad de Cuenca-Ecuador (Tesis de maestría en Sistemas de Información Geográficas). Universidad San Francisco de Quito, Quito. 
(Des)conexión durante el viaje turístico: uso de smartphones por parte de viajeros independientes en la ciudad de Guayaquil

McCabe, S. (2002). The tourist experience and everyday life. En G. Dann, The Tourist as a Metaphor of the Social World (pp. 61-75). Oxon: Cabi.

Miles, M., Huberman, A. y Saldaña, J. (2014). Qualitative Data Analysis: A Methods Sourcebook. Thousand Oaks: Sage.

Moores, S. (2005). Media/Theory: Thinking about Media and Communications. Abingdon: Taylor \& Francis.

mobiThinking. (2014). Global mobile statistics 2014. En mobiForge. Recuperado de https://mobiforge.com/research-analysis/global-mobile-statistics-2014-home-all-latest-stats-mobile-web-apps-marketingadvertising-subscriber [2016, 6 de abril].

Munar, A. M. y Jacobsen, J. K. S. (2014). Motivations for sharing tourism experiences through social media. Tourism Management, 43, 46-54.

Okazaki, S., Campo, S., Andreu, L. y Romero, J. (2015). A latent class analysis of Spanish travelers' mobile Internet usage in travel planning and execution. Cornell Hospitality Quarterly, 56(2), 191-201.

Pew Research Center. (2016). Mobile fact sheet. En Pew Research Center. Recuperado de http://www.pewinternet.org/fact-sheets/mobile-technology-fact-sheet/ [2016, 7 de abril].

Robson, C. y McCartan, K. (2016). Real World Research. Oxford: Wiley.

Think Google (Tns/Google). (2012). Our Mobile Planet: Argentina, Cómo entender a los usuarios de celulares. En SlideShare. Recuperado de http://www.slideshare.net/Menemadrid/our-mobile-planet-argentina [2016, 6 de abril].

Tussyadiah, I. P. y Fesenmaier, D. R. (2009). Mediating tourist experiences: Access to places via shared videos. Annals of Tourism Research, 36(1), 24-40.

Tussyadiah, I. P., \& Zach, F. J. (2012). The role of geo-based technology in place experiences. Annals of Tourism Research, 39(2), 780-800.

TripAdvisor. (2015). Trip Barometer March 2015. Global Travel Economy Report. Recuperado de https://d2bxpc4ajzxry0.cloudfront.net/TripAdvisorInsights/sites/default/files/downloads/ajax/tripbarometermarch2015_ globalreport_us.pdf [2016, 6 de abril].

unwTo. (2016). unwTo World Tourism Barometer, January. Recuperado de http:// 
www.capetown.travel/uploads/files/UNWTO_World_Tourism_ Barometer_January_2016.pdf [2016, 7 de abril].

Vallespín Arán, M., Molinillo, S. y Muñoz-Leiva, F. (2015). Perfil del consumidor turístico móvil: Una aproximación al usuario experimentado. Dos Algarves: A Multidisciplinary E-Journal, 26(1), 27-46.

Wang, D., Park, S., \& Fesenmaier, D. (2011). An examination of information services and smartphone applications. Memorias de la Graduate Student Research Conference in Hospitality and Tourism, enero 6-8 del 2011 en Houston, Texas. Amherst: University of Massachusetts.

Wang, D. y Xiang, Z. (2012). The new landscape of travel: A comprehensive analysis of smartphone apps. En M. Fuchs, F. Ricci y L. Cantoni (eds.), Information and Communication Technologies in Tourism 2012 (pp. 308-319). Viena: Springer.

Wang, D., Park, S. y Fesenmaier, D. R. (2012). The role of smartphones in mediating the touristic experience. Journal of Travel Research, 51(4), 371-387.

Wang, D. y Fesenmaier, D. R. (2013). Transforming the travel experience: The use of smartphones for travel. En L. Cantoni y Z. Xiang (eds.), Information and Communication Technologies in Tourism 2013 (pp. 58-69). Berlín: Springer.

Wang, D., Xiang, Z. y Fesenmaier, D. R. (2014). Adapting to the mobile world: A model of smartphone use. Annals of Tourism Research, 48, 11-26.

Wang, D., Xiang, Z. y Fesenmaier, D. R. (2016). Smartphone use in everyday life and travel. Journal of Travel Research, 55(1), 52-63. 
